\title{
An Enhanced Framework for Sentiment Analysis of Students' Surveys: Arab Open University Business Program Courses Case Study
}

\section{Mohammed Atif*}

Department of Computer and Systems Engineering, Alexandria University, Alexandria, Egypt

*Corresponding author: Mohammed Atif, Department of Computer and Systems Engineering, Alexandria University, Alexandria, Egypt, Tel: +20 3 5921675; E-mail: eng.mohamedatif@gmail.com

Received date: October 17, 2017, Accepted date: January 23, 2018, Published date: January 30, 2018

Copyright: (c) 2018 Atif M. This is an open-access article distributed under the terms of the Creative Commons Attribution License, which permits unrestricted use, distribution, and reproduction in any medium, provided the original author and source are credited.

\begin{abstract}
We present an enhanced framework for sentiment analysis which can be used for universities to improve student retention, teaching, and facilities. In addition, our proposed framework can be an important source for further analysis and improved decision-making. To best of our knowledge, this is the first work which targets student comments within surveys. We believe that students' comments are a good source to capture the overall students' sentiment. Our framework shows 0.8 accuracy when using 4 grams.
\end{abstract}

Keywords: Sentiment analysis; Comments; Survey; Decision making; Framework

\section{Introduction}

Student survey is a crucial tool to improve universities teaching and facilities. Many universities including Arab Open University (AOU) are conducting a mandatory survey every semester to get students' opinions/voice using Learning Management System (LMS).

The survey designed to have two kinds of questions as follows:

- Interval Scale Question (ISQ)

- Open-ended Question/Comment.

Using ordinary analytical tools, it is simple to provide a summary of students' opinions/views from ISQ type of questions. As students' comments are not quantitative data, it is very hard to analyze them, and it requires time and experts to do so.

Sentiment Analysis (SA) is the process of computationally/ statistically identifying and categorizing opinions expressed in a piece of text, especially to determine whether the writer's attitude towards a subject, topic, category, etc. is positive, negative.

Using SA, an enhanced framework for AOU is proposed to provide analysis for students' comments. Our objective is to the analysis of student's comments using SA technique to improve teaching and learning facilities of $\mathrm{AOU}$ which enhance/improve student retention.

The remainder of the paper is organized as follows. We discuss existing sentiment analysis methods and techniques. We present our framework for sentiment analysis of students' surveys and experiments. Finally, conclusion and future work are discussed.

\section{Existing Sentiment Analysis Methods and Techniques}

The processes of sentiment analysis differ from system to system based on 1) types of the classes to predict (positive or negative, subjective or objective), 2) and different levels of classification (sentence, phrase, or document level) [1-3]. In addition, sentiment analysis differs in terms of language that is processed. The authors
[4-6] proposed a system for subjectivity and sentiment analysis (SSA) for Arabic social media genres. The system deals with Arabic rich language which has significant complexities than the English language.

Recently, social networks become popular including Facebook, twitter, etc. and they become an emerging challenging sector where the natural language expressions of people can be easily reported through short but meaningful text messages. Many types of research proposed techniques for social networks sentiment analysis [7-12]. The main objectives of social networks sentiment analysis are to better understand consumer's feelings towards a brand, deliver signals into shifts in a brand and provide a better understanding of how a product or brand is perceived compared to the competition.

Although there are many different classifications for sentiment analysis, they are based on the same concept. Figure 1 shows overall framework. There is a training set which is used to learn the classifier. After building the classifier, a test set is used to check the accuracy of the classifier.

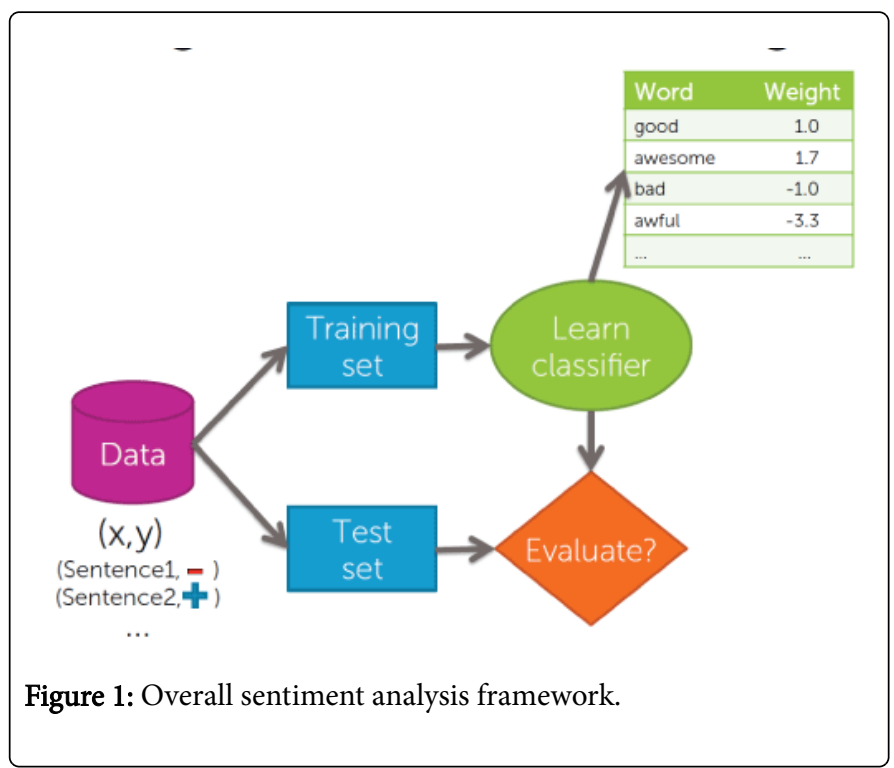


Page 2 of 3

The process of prediction the sentiment is shown in Figure 2 where a sentence comes from a review and the classifier classifies the document whether positive or negative sentiment.

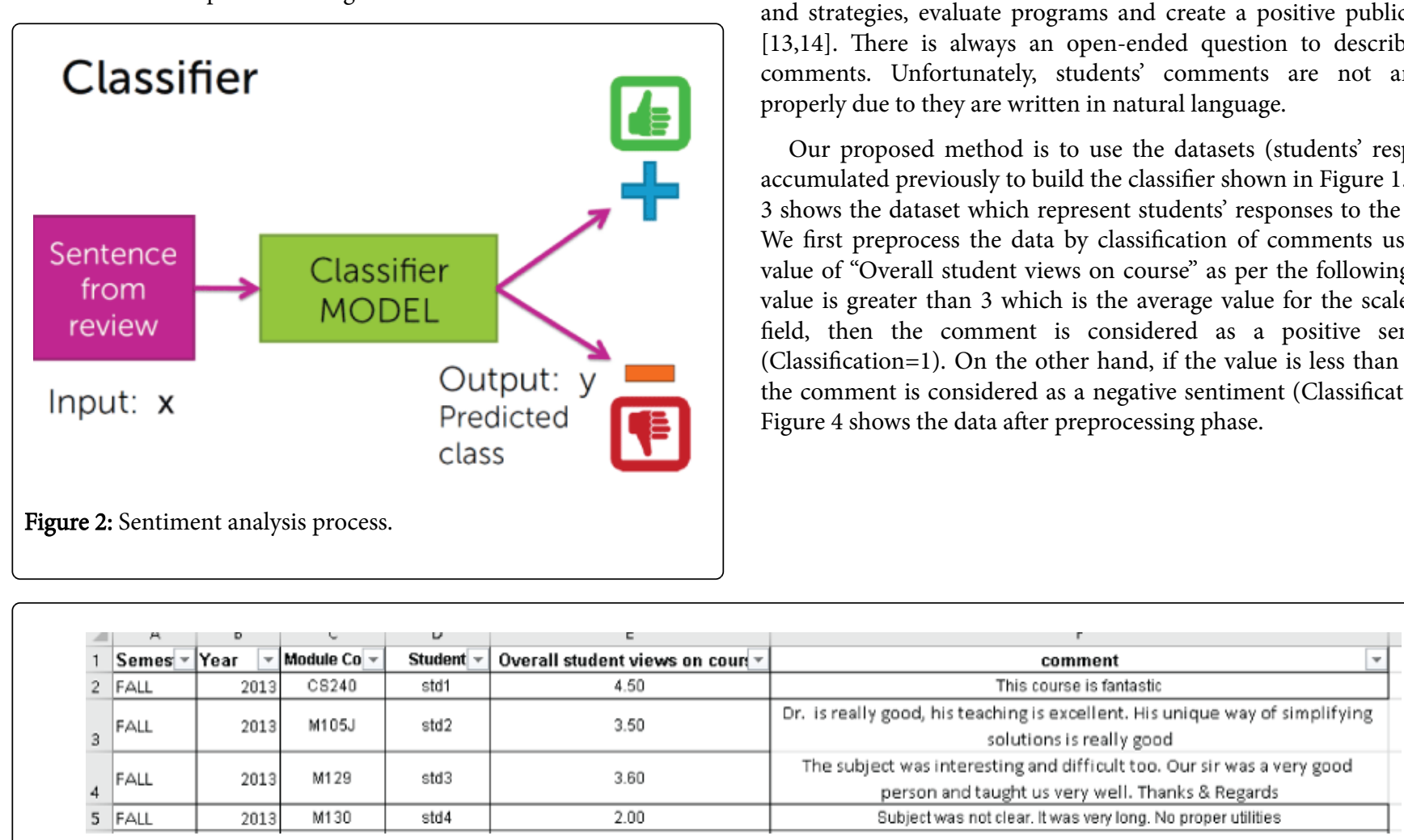

Figure 3: Survey responses.

\section{Proposed Framework}

Most of the universities provide surveys to students to develop goals and strategies, evaluate programs and create a positive public image $[13,14]$. There is always an open-ended question to describe their comments. Unfortunately, students' comments are not analyzed language. the comment is considered as a negative sentiment (Classification $=0$ ). Figure 4 shows the data after preprocessing phase.

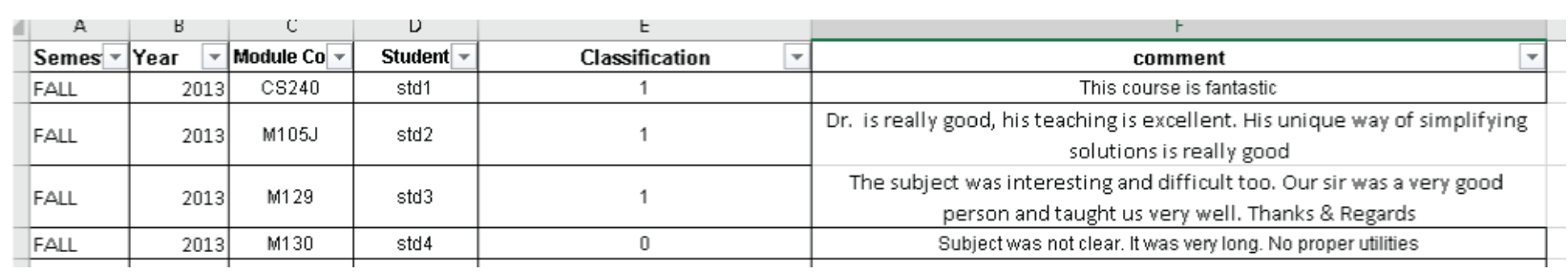

Figure 4: Dataset after classification of comments.

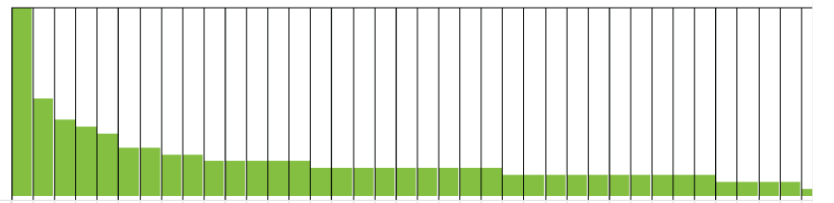

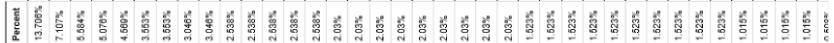

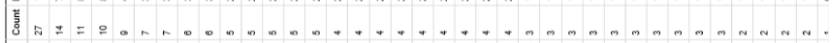

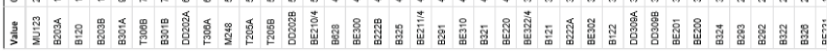

Figure 5: Contribution of different course in the dataset.
Our study is applied to the business program in AOU Kuwait branch. Figure 5 shows the course of the business program and their contributions in the dataset.

We used Graph lab library [15] to train the classifier and calculate accuracy. Table 1 shows the number of consecutive of words ( $\mathrm{N}$-grams) versus accuracy [16]. As we increase the $\mathrm{N}$-grams, the processing time increases and the accuracy increase, however at a certain point the accuracy saturates. We chose 4 grams as best setup for the classifier as it achieves the best accuracy with smallest $\mathrm{N}$ grams. 
Citation: Atif M (2018) An Enhanced Framework for Sentiment Analysis of Students' Surveys: Arab Open University Business Program Courses Case Study. Bus Eco J 9: 337. doi:10.4172/2151-6219.1000337

Page 3 of 3

\begin{tabular}{|l|l|}
\hline $\mathbf{N}$-gram(s) & Accuracy \\
\hline 1 gram & 0.5 \\
\hline 2 grams & 0.65 \\
\hline 3 grams & 0.72 \\
\hline 4 grams & 0.8 \\
\hline 5 grams & 0.8 \\
\hline
\end{tabular}

Table 1: Accuracy for different N-gram(s).

\section{Conclusion and Future Work}

We presented an enhanced framework for sentiment analysis which can be utilized for universities. We applied our framework for AOU Kuwait branch especially business program. We studied different settings for $\mathrm{N}$ grams, we found that 4 -grams is the best setting in terms of performance and accuracy.

In this framework, we did not spend much effort in studying other programs which contains Arabic comments [16]. It would be extremely interesting to extend the framework to include Arabic comments. It requires much more efforts as Arabic is written from right to left and there is no capitalization. Also, letters change their shape according to their position.

\section{References}

1. Michelle A, Kondrak G (2008) A comparison of sentiment analysis techniques: Polarizing movie blogs. Advances in Artificial Intelligence, pp: 25-35.

2. Punneliparambil ARG (2017) Latest trends in sentiment analysis-A survey. International Journal of Computer Science and Engineering Communications 5: 1606-1611.

3. Sara R, Farra N, Nakov P (2017) SemEval-2017 task 4: Sentiment analysis in Twitter. In: Proceedings of the 11th International Workshop on Semantic Evaluation (SemEval-2017), pp: 502-518.

4. Amira S, Rafea A (2012) Sentence-level Arabic sentiment analysis In Collaboration Technologies and Systems (CTS), 2012 International Conference, pp: 546-550.

5. Ahmed M, Darwish K (2013) Subjectivity and Sentiment Analysis of Modern Standard Arabic and Arabic Microblogs. In WASSA@ NAACLHLT, pp: 55-64.
6. Mageed MA, Diab M, Kübler S (2014) SAMAR: Subjectivity and sentiment analysis for Arabic social media. Computer Speech \& Language 28: 20-37.

7. Alec G, Huang L, Bhayani R (2009) Twitter sentiment analysis. Entropy 17: 252.

8. Alvaro O, Martín JM, Carro RM (2014) Sentiment analysis in Facebook and its application to e-learning. Computers in Human Behavior 31: 527-541.

9. Chenhao T, Lee L, Tang J, Jiang L, Zhou M et al., (2011) User-level sentiment analysis incorporating social networks. In Proceedings of the 17th ACM SIGKDD international conference on Knowledge discovery and data mining, pp: 1397-1405.

10. Adam B, Conway M, McInerney L, O'Hare N, Smeaton AF (2009) Combining social network analysis and sentiment analysis to explore the potential for online radicalisation. ASONAM '09 Proceedings of the 2009 International Conference on Advances in Social Network Analysis and Mining, pp: 231-236.

11. Georgios P, Thelwall M (2017) Sensing Social Media: A Range of Approaches for Sentiment Analysis. Cyberemotions, pp: 97-117.

12. Linda PH, Evans AT, Nickell L, Reboli AC, Coplit LD et al., Assessing the learning environment for medical students: an evaluation of a novel survey instrument in four medical schools. Academic Psychiatry 41: 354-359.

13. Mark LA, Scott N, Partington S, Oczujda A (2017) Coherence between text comments and the quantitative ratings in the UK's National Student Survey. Journal of Further and Higher Education 41: 16-29.

14. Yucheng L, Gonzalez JE, Kyrola A, Bickson D, Guestrin CE, et al., (2014) Graphlab: A new framework for parallel machine learning.

15. Ahmed N, Dinçer K, Sever H (2016) Investigation of the Feature Selection Problem for Sentiment Analysis in Arabic Language. Research in Computing Science 110: 41-54.

16. https://www.knime.com/blog/sentiment-analysis-with-n-grams. 